\title{
Illustrating Multipath Propagation by Means of Sound Waves
}

\author{
Luis Mendo \\ Computer Engineering Dept., Autonomous University of Madrid; \\ and Signals, Systems and Radiocommunications Dept., Polytechnic University of Madrid \\ E-mail: Imendo@grc.ssr.upm.es
}

\begin{abstract}
A method is presented that uses sound waves to illustrate multipath-propagation concepts. This is based on the existing analogy between radio and sound waves, and has the advantage that the required equipment is easily available. Different measurement techniques are introduced to respectively examine time dispersion, time variation, and both aspects of the multipath channel simultaneously. The proposed techniques are justified, example results are given, and the specific features that are illustrated by each type of measurement are discussed. Suggestions are given regarding application in courses on wireless communications.
\end{abstract}

Keywords: Communication engineering education; multipath channels; fading channels; dispersive channels; time-varying channels; acoustic propagation; electromagnetic propagation; electrical engineering education

\section{Introduction}

$\mathbf{E}$ ducators teaching electrical-engineering courses teach concepts for which concrete examples are often difficult to give. In particular, the theory of electromagnetic fields and radiowave propagation are based on ideas that the students may find difficult to grasp. Real-life examples are not abundant, and remain as somewhat unfamiliar constructions, since radio waves are not directly detected by our senses. The fact that light wavelengths are so small implies that in our daily experience with light, we do not normally detect effects such as diffraction or destructive interference, which, nevertheless, are very important in radio propagation. Analogies with light, therefore rather than helping, can be detrimental, making the referred-to effects appear as counterintuitive.

One of the fundamental aspects of radio communication is multipath propagation. Understanding this issue requires modeling the propagation channel as a linear time-variant system, characterized by its low-pass equivalent time-dependent impulse response. Throughout the paper, given a carrier frequency $f_{0}$, a bandpass signal will be denoted as $\tilde{x}(t)$, and its low-pass equivalent signal as $x(t)$. Thus,

$$
\tilde{x}(t)=\operatorname{Re}\left[x(t) e^{\jmath 2 \pi f_{0} t}\right]
$$

Let $\tilde{s}(t)$ and $\tilde{r}(t)$ respectively denote the transmitted and received bandpass signals, with low-pass equivalent signals $s(t)$ and $r(t)$. The time-dependent impulse response of the low-pass equivalent channel, $h(t, \tau)$, is defined by the relationship

$$
r(t)=\int_{-\infty}^{\infty} s(t-\tau) h(t, \tau) d \tau
$$

The time-dependent impulse response is difficult to visualize, because of the existence of two time variables.

To achieve a thorough understanding of multipath, it is important to have the possibility of "seeing" the propagation channel at work, i.e., transmitting a signal and observing the received signal. Although this can be accomplished using adequate radio equipment, the availability of such equipment may be limited because of its cost.

This paper proposes a simple and inexpensive method to visualize the different aspects of multipath propagation, using sound instead of radio waves. This is based on the fact that radio propagation has many characteristics in common with that of sound waves: in particular, the existence of multipath. It should be noted that an exact characterization of a given propagation channel is not necessary (nor is it particularly enlightening). Rather, the interest is in "aggregate" results, related to the general properties of the channel. Although the exact characterization (impulse response) of radio and sound propagation channels in the same environment will normally differ, the general properties are analogous in both cases.

The general idea of the measurements is to transmit a known signal, observe the received signal, and compare both to extract information about the low-pass equivalent channel response $h(t, \tau)$. The reception is carried out using a matched filter or correlator, which maximizes the received signal-to-noise ratio in the presence of additive white Gaussian noise [1, Sec. 5.1]. Different 
choices of the transmitted signal allow observing different aspects of the impulse response: namely, the time-dispersion characteristics of the channel, related to the variable $\tau$ in $h(t, \tau)$, or the timevarying behavior, which corresponds to the variable $t$.

The necessary equipment is described in Section 2 . The experiments are organized into three sections: time-dispersion measurements (Section 3), time-variation measurements (Section 4), and simultaneous time-dispersion and time-variation measurements (Section 5). Suggestions on the application to a course on wireless communications are given in Section 6. Conclusions are summarized in Section 7.

Although all the techniques to be described can provide amplitude and phase information, only the former will be considered, i.e., $|h(t, \tau)|$, as this is deemed to be sufficiently descriptive while simplifying the representation.

\section{Equipment}

The transmitted signal is generated in a computer and sent to a loudspeaker. The received signal is recorded by another computer using a microphone. It is recommended that two different computers be used, as many sound cards have a non-negligible electrical coupling from output to input. If the measurement is done with only one computer, the impulse response will contain an artificial peak caused by this coupling.

At the transmitter, an external, amplified loudspeaker is preferred, as built-in loudspeakers tend to have poor response at low frequencies. Inexpensive, computer-oriented speakers are perfectly adequate. Since a pair of loudspeakers is normally used, the two of them should be placed together, or the balance control should be set so that only one produces sound. Signal generation and receiver processing can be carried out with $M A T L A B$ or similar software. In the following, it is assumed that MATLAB is used. Transmission power is usually not an issue, as most amplified speakers are capable of delivering more sound power than is required for the measurements.

For the receiver, an external microphone is preferable, as this facilitates its displacement during the measurement. An inexpensive microphone, such as those intended for general computer use, is sufficient. Either omnidirectional or directional microphones may be employed. Nonetheless, if a highly directional microphone is used, this should be taken into account when interpreting the measurement. The signal picked up by the microphone is recorded and saved to a sound file, using any audio-recording program. The sound file is then read by MATLAB for receiver processing.

All the operations to be described have been implemented by the author in $M A T L A B$. The programs are available upon request.

\section{Time-Dispersion Measurement}

The purpose of this measurement is to illustrate the time dispersion caused by the propagation channel. Aspects related to time variation are not considered, that is, the channel is assumed to be time-invariant. This requires that the equipment and the objects in the environment remain still while the transmission is taking place.
The channel is characterized by a time-invariant impulse response, $h(\tau)$, and Equation (1) reduces to

$$
r(t)=s(t) * h(t)
$$

where "*" denotes convolution. Time dispersion is thus described by $h(\tau)$. A less-informative description can be given in terms of the delay spread of the channel [2, Sec. 2.3.2].

It will be assumed that $h(\tau)=0$ for $\tau<0$ (causality), and for $\tau$ exceeding a certain value, $\tau_{\max }$, which represents an upper bound on the delays introduced by the channel.

\subsection{Signal Structure and Receiver Principle}

The reliability with which a signal can be detected against noise can be characterized by the ratio of signal energy, $E_{s}$, to noise-power spectral density, $N_{0}$. The signal energy is expressed as $P T$, where $P$ denotes received signal power, and $T$ is the integration time. In order to simultaneously achieve high resolution and good sensitivity, pulse-compression techniques can be applied [3, Sec. 11.5]. In particular, direct-sequence spread-spectrum (DSSS) signals can be used. The low-pass equivalent transmitted signal is thus

$$
s(t)=\sum_{i=i_{\min }}^{i_{\max }} S[i] g\left(t-i T_{c}\right),
$$

where $g(t)$ is the low-pass equivalent chip pulse, and $S[i]$ is a complex chip sequence. In this model, $i_{\text {min }}$ and $i_{\max }$ may be either finite or infinite. For simplicity, $g(t)$ is restricted to be a real function. It is also assumed to be zero outside $\left[0, T_{c}\right]$, i.e., non-overlapping, time-limited chip pulses are considered. With this approach, sensitivity can be improved by using a long signal and a large integration time, $T$. Resolution is determined by the chip duration, $T_{c}$, provided that the chip sequence has adequate autocorrelation properties, namely, a large sidelobe level (SLL), defined as the ratio of the autocorrelation value for zero lag to the maximum absolute value for nonzero lags.

Since only amplitude information is sought, the receiver should carry out a non-coherent correlation of the received signal. A natural approach is to use $i_{\min }=0, i_{\max }=M-1$, i.e., a chip sequence of finite length $M$, and to correlate the received signal with the complete transmitted signal. The integration time, $T$, equals the duration of the transmitted signal, $M T_{c}$. The ability to discriminate among paths with different delays depends on the aperiodic autocorrelation of the transmitted sequence, as well as on $T_{c}$.

Another possible approach is to use a periodic transmitted signal, obtained from repetitions of a chip sequence of finite length $M$, and to correlate the received signal with a single period of the transmitted signal. In practice, the periodic signal may be substituted for by a cyclically extended version, with $i_{\min }=-m_{1}$, 
$i_{\max }=M+m_{2}-1$, for $m_{1}, m_{2}$ suitably selected. The integration time, $T$, corresponds to the period $M T_{c}$, and it is the periodic autocorrelation of the sequence that determines performance.

The two approaches are analyzed in the Appendix. For the present case, a periodic (cyclically extended) design, using an m-sequence, is preferred. This implies that $\tau_{\max }$ should not exceed $M T_{c}$ in order to avoid ambiguity, and that the representation needs to be windowed, with a window length of $M T_{c}$. See Sections 9.2 and 9.4 in the Appendix for the details.

A rectangular chip pulse will be used. Small values of $T_{c}$ are desirable in order to obtain good resolution, but the allowed values are limited by the available bandwidth. The usable bandwidth for audio transmission is assumed to be from a few hundreds of $\mathrm{Hz}$ to several $\mathrm{kHz}$. These conservative values assure that the frequency response in the transmitting and receiving equipment is reasonably flat. According to this, the carrier frequency is limited to a few $\mathrm{kHz}$, and signal bandwidth should not exceed a value of the order of $1 \mathrm{kHz}$. Larger bandwidths can be used at the expense of some degradation in the measurement, as the "narrowband" assumption $f_{0} \gg 1 / T_{c}$ (see Section 9.1 in the Appendix) gradually fails to hold. With these constraints, $f_{0} \approx 3$ to $3.5 \mathrm{kHz}$, and $T_{c} \approx 0.7$ to $1.5 \mathrm{~ms}$, appear as reasonable choices. Given that sound propagates at a speed $V \approx 340 \mathrm{~m} / \mathrm{s}$ in air, the selected $T_{c}$ values correspond to path differences of 25 to $50 \mathrm{~cm}$.

\subsection{Receiver Processing Details}

Since the operations are performed on a computer, integration is substituted for by summation of discrete-time, quantized signals. Sample rates $f_{\mathrm{s}}$ of 44.1 or $48 \mathrm{kHz}$, which are standard values in digital audio [4, Sec. 4.5], are sufficient. Quantization noise is not an issue with modern sound cards, which use at least 16 bits per sample.

The receiver computes the non-coherent correlation $z(\tau)$ from the bandpass received signal $\tilde{r}(t)$ (see Section 9.2 in the Appendix), using Equations (3) and (5). However, even though correlation is the optimum operation from the point of view of resulting signal-to-noise ratio, certain additional processing can be applied to improve other aspects of the estimation.

If the received signal, $\tilde{r}(t)$, contains a strong interference or noise at a certain frequency, $f_{i}$, the squaring operations involved in computing $z(\tau)$ may give rise to intermodulation products with $f_{0}$. In particular, a 50 or $60 \mathrm{~Hz}$ component, arising from mains electricity, is usually present, and may cause a visible ripple in $z(\tau)$, with a frequency close to $f_{0}$. To avoid this, it is convenient to filter the signal prior to the non-coherent correlation. The corresponding filter will be referred to as a pre-filter. A discrete-time Butterworth design of order 4 and a pass-band of $\left[f_{0}-2 T_{c}, f_{0}+2 T_{c}\right]$ gives good results. Although this pre-filter hardly affects the signal, an identical filter can be applied at the transmitter to ensure matched-filtering operation at the receiver.

Assuming that two separate computers are used (as recommended in Section 2), a difference between sound-card clock rates at the transmitter and receiver will exist. Discrepancies of 30 to $80 \mathrm{ppm}$ have been measured by the author. This implies that the pattern signals generated at the receiver have a slightly different carrier frequency than the received signal (the chip rate is also affected, but to a lesser extent, as it is smaller than the carrier frequency). For $T_{c} \approx 1 \mathrm{~ms}$ and $f_{0} \approx 3 \mathrm{kHz}$, an $\mathrm{m}$-sequence of $M=4095$ gives an integration time of roughly $4 \mathrm{~s}$, or 12000 carrier cycles. With a clock-rate difference of $50 \mathrm{ppm}$, this introduces a phase change of more than half a cycle during the integration time, which can severely degrade the autocorrelation properties.

To solve this, carrier recovery is necessary [1, Sec. 6.1.2]. A simplified, non-real-time form of recovery can be used, exploiting the fact that the whole received signal is available at the time of processing. The procedure consists of carrying out the correlation using different carrier frequencies, and selecting the frequency that yields the largest peak in $z(\tau)$, i.e., that which maximizes $\max _{\tau} z(\tau)$. Let the candidate frequencies be given as $(1+k \delta) f_{0}$, $k \in \mathbb{Z}$, where $\delta$ is a relative step. The latter should be selected such that $\delta f_{0}$ gives an accumulated phase change equal to a small fraction of a cycle, that is, $M T_{c} \delta f_{0} \ll 1$. If the integration time, $M T_{c}$, is small, a $\delta$ value of the order of the expected clock-rate difference (tens of ppm) may already fulfill the stated condition. In this case, frequency correction is not necessary (nor would it be reliable).

Table 1 indicates the restrictions on signal and receiver parameters.

\subsection{Outline of Program}

The described steps are summarized as follows.

\section{Time-Dispersion Measurement Procedure}

1. Transmitter:

1.1 Generate $s(t)$ from $m$-sequence as in

Section 9.2, and obtain transmitted signal $\tilde{s}(t)$.

1.2 Apply pre-filter to $\tilde{s}(t)$.

1.3 Play signal.

Table 1. The restrictions on parameters for a time-dispersion measurement.

\begin{tabular}{|l|l|}
\hline Low-pass equivalent model & $f_{0} \gg 1 / T_{c}$ \\
\hline Available bandwidth & $100 \mathrm{~Hz}<\mathrm{f}_{0}<10 \mathrm{kHz}$ \\
\hline Sample rate & $f_{s} \gg f_{0}$ \\
\hline Frequency correction & $\delta \ll 1 /\left(M T_{c} f_{0}\right)$ \\
\hline Time-invariance of the channel & During $\left(M+m_{1}+m_{2}\right) T_{c}$ \\
\hline Ambiguity in $\tau$ & $\tau_{\max }<M T_{c}$ \\
\hline Resolution in $\tau$ & $T_{c}$ \\
\hline Cyclic extension & $m_{1}, m_{2}>\tau_{\max } / T_{c}$ \\
\hline Integration time & $M T_{c}$ \\
\hline SLL & $M$ \\
\hline
\end{tabular}




\section{Receiver:}

2.1 Record signal, save as sound file and read received signal $\tilde{r}(t)$ from file.

\subsection{Pre-filter signal.}

2.3 Generate correlation patterns $\tilde{s}_{p}(t), \tilde{s}_{p}^{\prime}(t)$ as defined in Section 9.2, for different carrier frequencies within a given frequency span.

\subsection{Compute the non-coherent correlation $z(\tau)$} from Equations (3) and (5), for each receiver carrier frequency, and choose that which gives maximum peak.

2.5 Plot the windowed version of $z^{2}(\tau)$ as an estimation of $|h(\tau)|^{2}$.

\subsection{Example Results}

The described procedure has been tested in a variety of environments. The results presented in this section, as well as those in subsequent sections, have been obtained with two laptop computers, using their internal sound cards. Consumer-range external loudspeakers and an inexpensive external microphone with moderate directivity were employed.

Figure 1a displays the measured response in a laboratory room. The room size was $14 \times 8.5 \times 3 \mathrm{~m}$, and it was divided into three modules, with plenty of equipment and furniture. This gave a delay spread of the order of tens of ms. The measurement parameters were $f_{0}=3.53 \mathrm{kHz}, T_{c}=1.36 \mathrm{~ms}, f_{s}=44.1 \mathrm{kHz}, M=127$, and $m_{1}=m_{2}=114$, with a maximum measurable delay of $\tau_{\text {max }}=155 \mathrm{~ms}$. The condition for the frequency-correction step in this case was $\delta \ll 1640 \mathrm{ppm}$. Taking into account that the clockrate difference was of the order of tens of ppm, frequency correction was thus not applied. The figure represents $|h(\tau)|^{2}$, normalized to a maximum value of one. The observed graph was in accordance with the expected delay spread.

Figure $1 \mathrm{~b}$ corresponds to a building lobby of size $17.5 \times 14 \times 6.5 \mathrm{~m}$, in which there were three columns and several decorative objects. The transmitter and receiver were located far apart, without line of sight. This constituted a larger, more spacious environment, with a significant contribution from multiple reflections. The resulting channel was thus more dispersive, with delays of hundreds of $\mathrm{ms}$, as can be seen in the figure. The signal parameters were the same as in Figure 1a, except that $M$ was increased to 4095 to improve sensitivity and to allow detection of larger delays. $m_{1}=m_{2}$ were set to 1228 , for a maximum measurable delay of $1.67 \mathrm{~s}$. Frequency correction was necessary in this case, with $\delta \ll 50 \mathrm{ppm}$. A value of $\delta=5 \mathrm{ppm}$ was used.

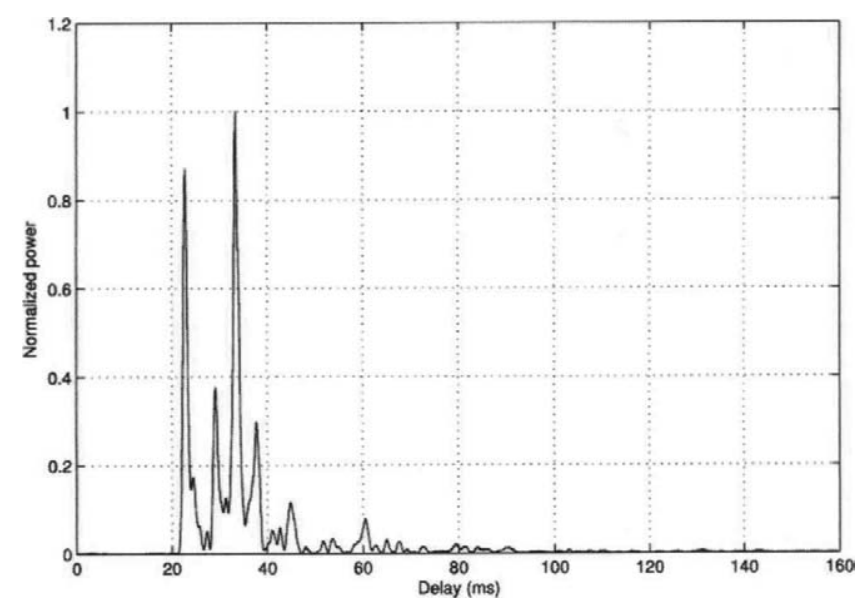

Figure 1a. An example of a time-dispersion measurement: an indoor environment with moderate dispersion.

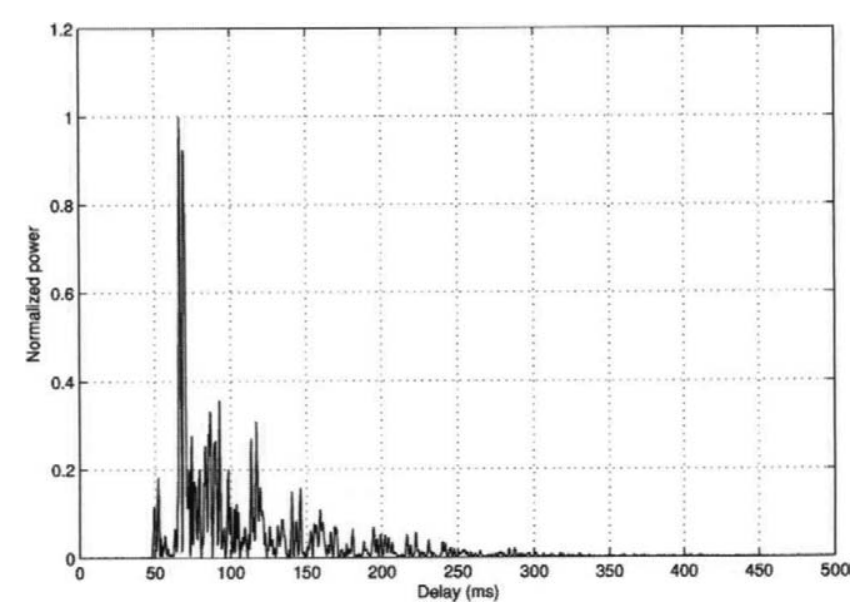

Figure 1b. An example of a time-dispersion measurement: a building lobby with large dispersion.

\subsection{Other Features that Can be Observed with this Measurement}

The measurement primarily serves to illustrate the timedispersion characteristics of the channel. The observed impulse response can be related to physical characteristics of the environment, such as room size, the amount of furniture, or the existence of line of sight.

As a byproduct, the existence of clock drift can be employed to demonstrate the importance of carrier recovery in communication systems. If the sequence length is gradually increased without applying frequency correction, the noise is first reduced due to larger integration time, but above a certain length, when the accumulated phase error is a significant fraction of a cycle, the measurement sharply degrades. 


\section{Time-Variation Measurement}

Time variation of the multipath channel manifests as fading, caused by constructive or destructive interference between different signal paths. The amount of time it takes for the fading level to change significantly is the coherence time of the channel.

The time-variation aspect of the channel is best observed by transmitting a long signal with constant amplitude, i.e., a sinusoid, $\tilde{s}(t)=\cos \left(2 \pi f_{0} t\right)$, and representing the amplitude variations in the received signal. The low-pass equivalent transmitted signal is $s(t)=1$, and thus Equation (1) reduces to

$$
r(t)=\int_{-\infty}^{\infty} h(t, \tau) d \tau
$$

This expression, in which the dependence on $\tau$ disappears by averaging, reflects the fact that time variations correspond to the $t$ variable in $h(t, \tau)$.

It will be assumed that the speed at which the receiver, transmitter, or any object in the environment moves is bounded by a certain value, $v_{\max }$.

\subsection{Signal Structure and Receiver Principle}

Since the transmitted signal is a sinusoid, and only amplitude information is desired, the optimum strategy is again a non-coherent correlator or matched filter. In this case, this is well approximated by a narrow bandpass filter tuned to $f_{0}$, followed by an envelope detector. This bandpass filter will be referred to as the receiving filter. Its output will be denoted as $\tilde{y}(t)$, and the output of the envelope detector will be denoted as $z(t)$.

The receiving filter helps remove noise and interference, and should be as narrow as possible. The received signal, $\tilde{r}(t)$, has a nonzero bandwidth as a result of Doppler spread [1, Sec. 14.1]. The maximum Doppler spread is $2 v_{\max } / \lambda$ [5, Sec. 3.2], where the wavelength, $\lambda$, is given as $V / f_{0}$. The filter bandwidth, $B$, should thus be slightly larger than $2 v_{\max } / \lambda$, in order to minimize noise while not altering the signal shape.

There is much freedom in the choice of $f_{0}$. Interestingly, values between $340 \mathrm{~Hz}$ and $3.4 \mathrm{kHz}$ give wavelengths of $0.1 \mathrm{~m}$ to $1 \mathrm{~m}$, which in radio correspond to the ultra-high-frequency (UHF) range.

\subsection{Receiver Processing Details}

The envelope detector can be realized by taking the absolute value of $\tilde{y}(t)$ and applying a low-pass filter, with cutoff frequency $f_{e}$ much smaller than $f_{0}$, to remove the variations at the carrier frequency. An alternative would be to dispense with the envelope detector and represent $\tilde{y}(t)$ : the envelope can be directly observed from the graph. In either case, in order to distinguish envelope variations from carrier variations, $f_{0}$ should be much greater than $v_{\max } / \lambda$, i.e., $v_{\max } \ll V$. This is clearly fulfilled with the displacement speeds occurring in practice. In the following, it will be assumed that an envelope detector is used, and the envelope obtained will be plotted in $\mathrm{dB}$.

The shape of the frequency response of the receiving filter is not very important, as long as it is approximately flat within the maximum Doppler spread. A Butterworth bandpass design of order two, for example, gives good results. Carrier recovery is not needed, as the frequency variations due to clock-rate differences are well within the filter bandwidth. For the low-pass filter of the envelope detector, a more-selective frequency response is advised, such as that obtained with a Butterworth filter of order four, in order to avoid visible ripples at $f_{0}$.

The restrictions on signal and receiver parameters are summarized in Table 2.

\subsection{Outline of Program}

\section{Time-Variation Measurement Procedure}

1. Transmitter:

1.1 Generate transmitted signal (sinusoid), $\tilde{s}(t)$.

1.2 Play signal.

2. Receiver:

2.1 Record signal, save as sound file, and read received signal $\tilde{r}(t)$ from file.

2.2 Filter $\tilde{r}(t)$ to obtain $\tilde{y}(t)$.

2.3 Obtain $z(t)$ as the envelope of $\tilde{y}(t)$.

2.4 Plot $z(t)$.

\subsection{Example Results}

Figure 2 shows measurements in an empty classroom of small size (a capacity of 24 students). Two different carrier fre-

Table 2. Restrictions on parameters for a time-variation measurement.

\begin{tabular}{|l|l|}
\hline Available bandwidth & $100 \mathrm{~Hz}<\mathrm{f}_{0}<10 \mathrm{kHz}$ \\
\hline Maximum displacement speed & $v_{\max } \ll V$ \\
\hline Sample rate & $f_{s} \gg f_{0}$ \\
\hline Receiving filter bandwidth & $B>2 f_{0} v_{\max } / V$ \\
\hline Envelope detector cutoff frequency & $v_{\max } / V<f_{e} / f_{0} \ll 1$ \\
\hline Integration time & $\approx 1 / B$ \\
\hline
\end{tabular}




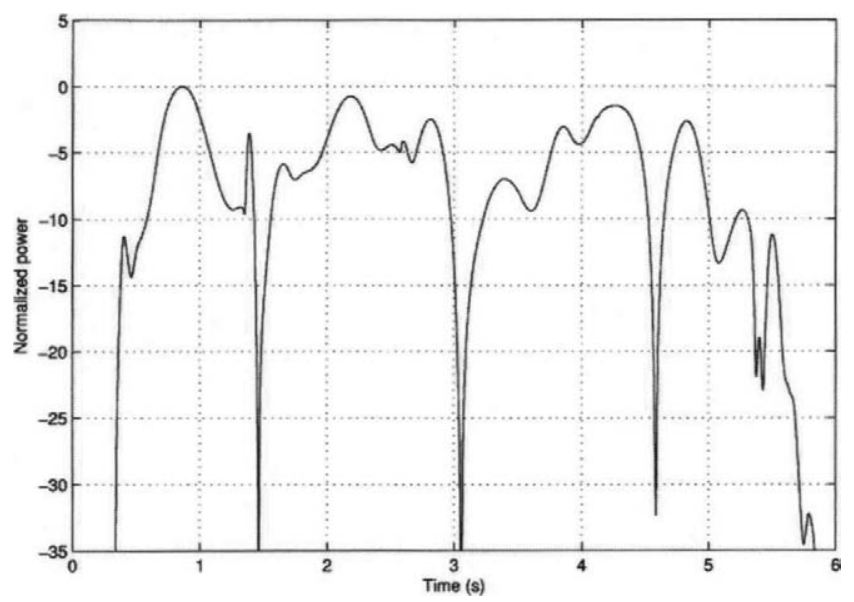

Figure 2a. An example of a time-variation measurement: carrier frequency $f_{0}=500 \mathrm{~Hz}$.

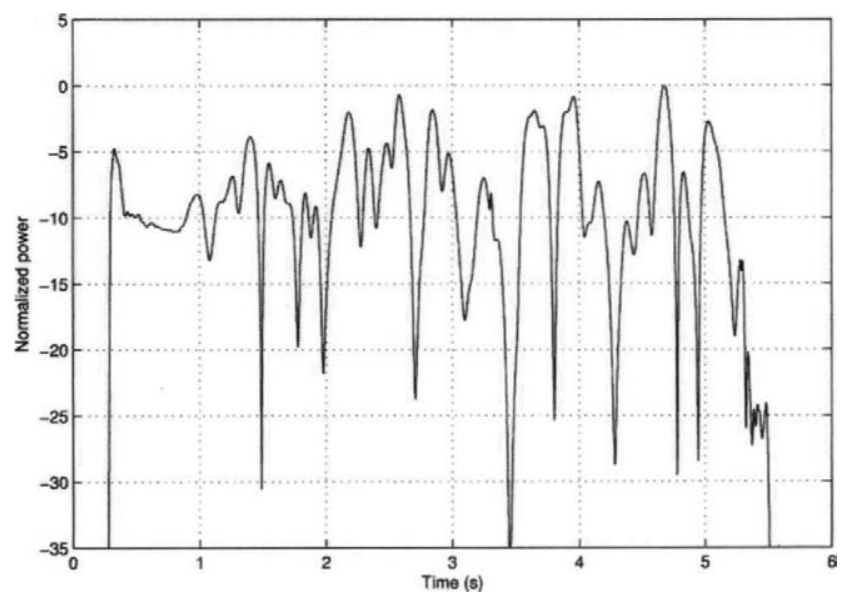

Figure 2b. An example of a time-variation measurement: carrier frequency $f_{0}=2 \mathrm{kHz}$.

quencies were used: $f_{0}=500 \mathrm{~Hz}$ in Figure 2a, and $2 \mathrm{kHz}$ in Figure $2 \mathrm{~b}$, with a signal duration of $5 \mathrm{~s}$. The sample rate was $f_{s}=48 \mathrm{kHz}$, and the receiving-filter bandwidth was $0.02 f_{0}$. In both cases, the microphone was moved at an approximate speed of $v=1 \mathrm{~m} / \mathrm{s}$. This gave a Doppler spread of $2 v / \lambda \approx 0.006 f_{0}$, which was within the filter pass-band. The cutoff frequency of the envelope detector was $50 \mathrm{~Hz}$. The received power was normalized to its maximum value. It was seen that even though the filter bandwidth was not tightly adjusted to that of the received signal, the filter removed a sufficient amount of noise to give an accurate representation up to the displayed $35 \mathrm{~dB}$ range. In fact, noise did not appear in the graph within the two small intervals before and after the signal was present, which indicated that it was below the $-35 \mathrm{~dB}$ relative level.

In the figure, the length of the received signal was seen to be slightly greater than $5 \mathrm{~s}$ : this was due to time dispersion. The envelope variations had the typical aspect of multipath fading, with fades of more than $30 \mathrm{~dB}$. The coherence time could be computed as the inverse of the Doppler spread, i.e., $\lambda /(2 v)$. With $v=1 \mathrm{~m} / \mathrm{s}$, this gave $0.34 \mathrm{~s}$ for $f_{0}=500 \mathrm{~Hz}$, and $0.085 \mathrm{~s}$ for $f_{0}=2000 \mathrm{~Hz}$, which was in agreement with the graphs.

\subsection{Other Features that Can be Observed with this Measurement}

The measurement procedure described is intended to illustrate time-varying fading, and the relationship between displacement speed, carrier frequency, and coherence time. Additionally, frequency selectivity could be demonstrated by simultaneously transmitting at two different frequencies and comparing the results.

As a side issue, the measurement can illustrate noise removal in the received signal, thanks to matched filtering. By using different receiving-filter bandwidths, the improvement of signal-to-noise ratio can be made evident, with larger bandwidths resulting in noisier representations. This is best viewed by keeping the microphone and environmental objects still, so that the signal envelope is constant. Note that this effect is particularly easy to observe here, because - unlike in the time-dispersion measurement - the transmitted signal is a sinusoid, and the receiving operations do not alter its shape.

\section{Simultaneous Time-Dispersion and Time-Variation Measurement}

While Sections 3 and 4 addressed the time-dispersion and time-variation aspects, respectively, a complete characterization of the multipath channel requires dealing with both simultaneously, i.e., observing the full time-variant impulse response, $h(t, \tau)$. As before, for the sake of simplifying representation, only its amplitude, $|h(t, \tau)|$, will be considered.

As in previous sections, it will be assumed that $h(t, \tau)=0$ for $\tau$ outside $\left[0, \tau_{\max }\right]$, and that movement speed does not exceed $v_{\max }$.

\subsection{Signal Structure and Receiver Principle}

The measurement follows a similar procedure to that in Section 3, only repeated many times to capture time variation. The low-pass equivalent transmitted signal, $s(t)$, thus consists of a basic signal, $s_{r}(t)$, which is repeated $R$ times with period $T_{r}$. This stems of the fact that an ambiguity equal to $T_{r}$ is introduced into the measured delays. As detailed in Section 9.5 in the Appendix, an aperiodic design, based on polyphase Barker sequences, is more suited to this measurement. This implies that $s_{r}(t)$ consists of a short active part, $s_{a}(t)$, of duration $T_{a}=M T_{c}$, where $M$ is the sequence length, followed by a gap of length $T_{r}-T_{a}$. The integration time equals $T_{a}$. The value $T_{a}$ must be chosen such that the channel is approximately invariant during this time. Furthermore, $T_{r}$ should be such that the channel variation is reasonably smooth during this period. The chip period and pulse shape are as in Section 3.1. 


\subsection{Receiver Processing Details}

The receiver is similar to that described in Section 3.2, except that the effect of clock drift is different, and additional processing is needed to produce a graphical representation that includes time variation as well as time dispersion. This is discussed in the following.

Because the integration time is small, a correction of the carrier frequency is not needed. However, since the measurement is repeated for a total time $R T_{r}$, the difference in clock rates may give a significantly large accumulated error in the measured delays. For example, if $R T_{r}=20 \mathrm{~s}$, a $50 \mathrm{ppm}$ difference gives a $1 \mathrm{~ms}$ error between delays, measured at the beginning and at the end of the observation time. This error is of the order of $T_{c}$, and thus noticeable. To correct this, the received signal must be re-sampled. The adequate re-sampling ratio can be determined by carrying out a long measurement of an invariant channel, and choosing the ratio that gives constant delays for the detected paths. If the time-dispersion measurement has been previously performed, an alternative is to apply the same ratio as obtained for the carrier frequency in that case.

The function $|h(t, \tau)|^{2}$ is best displayed as an image, with the function value in $\mathrm{dB}$ represented by different colors or grey levels. In the following, it will be assumed that $t$ is represented on the horizontal axis, and $\tau$ is on the vertical axis. The representation range for the function values should be slightly smaller than the sidelobe level, so that most spurious peaks caused by secondary lobes are not shown. The reason is that when a path is detected as an autocorrelation peak, all other paths produce interference through autocorrelation secondary lobes. The fact that these paths simultaneously interfere with the observed path gives rise to an interference level that may be above the sidelobe level.

To compose the image, the domain of $z(\tau)$ is partitioned into intervals of length $T_{r}$, i.e.,

$$
\left[t_{0}+i T_{r}, t_{0}+(i+1) T_{r}\right), i \in \mathbb{Z},
$$

where $t_{0}$ is a time reference to be specified. This divides $z(\tau)$ into different segments $z_{i}(\tau)$, defined as

$$
z_{i}(\tau)=z\left(\tau+t_{0}+i T_{r}\right), \quad \tau \in\left[0, T_{r}\right) .
$$

Each segment is displayed as a vertical line in the image, with successive values of $i$ corresponding to consecutive lines. The time reference, $t_{0}$, should be chosen such that each of the measured responses, $\left|h\left(t_{i}, \tau\right)\right|$ is contained in a vertical line of the image, i.e., it is not split between consecutive lines. The adjustment can be done automatically, exploiting the fact that in most channels, received power tends to decrease with $\tau$. Thus, $\max _{\tau} \sum_{i} z_{i}^{2}(\tau)$ is computed, and $t_{0}$ is set so that this maximum is located near the bottom of the image.

Another issue with the image obtained concerns its horizontal and vertical resolutions. The number of horizontal $(t)$ pixels is $R$, whereas the number of vertical $(\tau)$ pixels is $T_{r} f_{s}$. The latter is typically much larger than can fit on a screen, and the former may be too small for adequate representation. This can be fixed by down-sampling (with an anti-aliasing filter) in $\tau$, and up-sampling (with interpolation) in $t$.

Table 3 summarizes the restrictions on signal and receiver parameters.

\subsection{Outline of Program}

\section{Simultaneous Time-Dispersion and Time-Variation Measurement Procedure}

1. Transmitter:

1.1 Generate $s_{a}(t)$ and $s_{r}(t)$ from Barker sequence as in Section 9.5. Obtain $s(t)$ and $\tilde{s}(t)$.

1.2 Apply pre-filter to $\tilde{s}(t)$.

1.3 Play signal.

2. Receiver:

2.1 Record signal, save as sound file, and read received signal, $\tilde{r}(t)$, from file.

\subsection{Resample received signal.}

2.3 Pre-filter signal.

2.4 Generate correlation patterns, $\tilde{s}_{a}(t), \tilde{s}_{a}^{\prime}(t)$ from $s_{a}(t)$, as in Section 9.1 .

2.5 Compute non-coherent correlation, $z(\tau)$, as in Equations (2) and (3), assuming equal received and transmitted carrier frequencies.

2.6 Compose image, adjust horizontal and vertical resolutions, and display.

Table 3. Restrictions on parameters for a simultaneous timedispersion and time-variation measurement.

\begin{tabular}{|l|l|}
\hline Low-pass equivalent model & $f_{0} \gg 1 / T_{c}$ \\
\hline Available bandwidth & $100 \mathrm{~Hz}<\mathrm{f}_{0}<10 \mathrm{kHz}$ \\
\hline Sample rate & $f_{s} \gg f_{0}$ \\
\hline Local time-invariance & $v_{\max } \ll V /\left(f_{0} M T_{c}\right)$ \\
\hline Time variation & $v_{\max } \approx V /\left(f_{0} T_{r}\right)$, or smaller \\
\hline Resolution in $t$ & $T_{r}$ \\
\hline Ambiguity in $\tau$ & $\tau_{\max }<T_{r}$ \\
\hline Resolution in $\tau$ & $T_{c}$ \\
\hline Integration time & $M T_{c}$ \\
\hline SLL & $M$ \\
\hline
\end{tabular}




\subsection{Example Results}

It is recommended that the measurements be taken in environments with little or moderate time dispersion. A large $\tau_{\max }$ requires a correspondingly large $T_{r}$, which in turn reduces $v_{\max }$. Delay spreads that are too large might thus require impractically slow movements. In addition, it is best to choose an environment that is not very cluttered. If there are many paths with similar delays, the representation becomes too "tangled," making it difficult to identify paths.

Figure 3 shows two measurements in the same indoor setting as in Figure 1a (a laboratory room with a low delay spread). The signal parameters were $f_{0}=2.94 \mathrm{kHz}, \quad T_{c}=0.68 \mathrm{~ms}$, $f_{s}=44.1 \mathrm{kHz}$, a binary Barker sequence with $M=13$, $T_{r}=113 T_{c}=77 \mathrm{~ms}$, and $R=180$. The selected $T_{c}$ gave a delay resolution around $1 \mathrm{~ms}$, and the signal length, $R T_{r}$ was $13.84 \mathrm{~s}$. The origin of the vertical axis was arbitrary (as stems from Section 5.2). The represented $|h(t, \tau)|^{2}$ was normalized, with $0 \mathrm{~dB}$ corresponding to the maximum value.

In Figure 3a, all objects in the environment were stationary, except that a metallic plate, situated behind the microphone, was initially still, then was slowly moved farther away, and then was still again. There was line of sight between the transmitter and receiver. All resolvable paths were initially seen as horizontal lines, corresponding to constant delays, but at $t \approx 1.5 \mathrm{~s}$, the delay of the second detected path began to increase. This path corresponded to the wave reflected in the moving plate, while the first path was the direct ray, the delay of which did not change. It is interesting to note how other paths also experienced changes as a consequence of the plate's motion. These paths corresponded to multiple reflections involving the plate and other objects in the environment.

Consider the path with a single reflection in the moving plate. As its delay approached that of some other path, both paths became indistinguishable for the correlator, and a characteristic interference pattern arose. This could be seen as a series of maxima and minima in the region where the lines described by the two paths crossed. This phenomenon was evident in Figure 3a for $t \approx 6.5$ to $8.5 \mathrm{~s}$. Similar patterns between other paths with larger delays were discernible, as well.

In Figure 3b, the microphone was in the line of sight from the loudspeaker, and it was hanging from its cable, oscillating like a pendulum along the direction from the loudspeaker. This gave an approximately sinusoidal variation in the delay of the direct path, as well as in several reflected paths, as was clearly seen in the image.

The figures obtained from the measurements presented in Sections 3 and 4 can be considered as particular cases of the type of representation described here. Namely, a time-dispersion measurement such as those in Figure 1 corresponds to a vertical cut of the $|h(t, \tau)|^{2}$ image. Similarly, the time-varying fading observed in Figure 2 is actually an interference pattern between irresolvable paths, analogous to that observed in Figure $3 \mathrm{a}$. The difference is that in the time-variation measurement, no attempt was made to resolve any paths, and thus all of them interfered, giving a more irregular pattern.

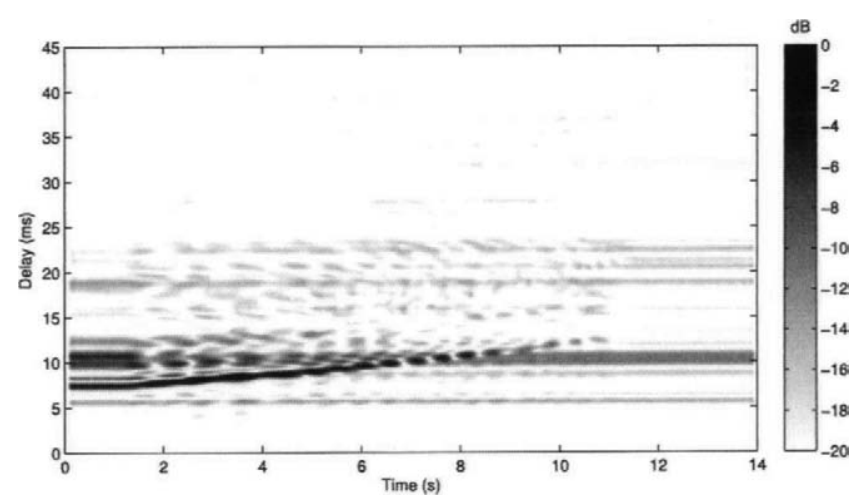

Figure 3a. An example of a simultaneous time-dispersion and time-variation measurement: a reflecting object moving away from the receiver.

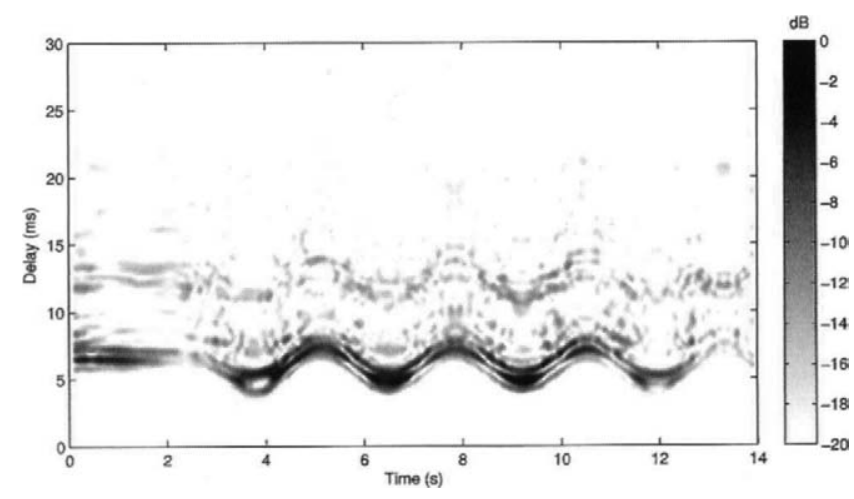

Figure 3b. An example of a simultaneous time-dispersion and time-variation measurement: the receiver position was oscillating along the direction from transmitter.

Even though the measurement procedure just presented is a generalization of the preceding procedures, this generalization must be understood as a compromise. There is a tradeoff involved: since more information is extracted from the received signal, the quality of this information is necessarily degraded. Specifically, the achievable $E_{s} / N_{0}$ and sidelobe levels were lower than in the measurements described in Section 3, as $M$ was here limited by the fact that the channel needed to be constant during the integration time. Likewise, the representation was noisier, and had poorer time resolution than, those of Section 4 . Consequently, the most appropriate type of measurement should be selected in each particular situation, depending on which aspects are deemed to be more important.

\subsection{Other Features that can be Observed with this Measurement}

Besides the time-dispersion and time-variation aspects, two other issues can be illustrated. First, the existence of a clock-rate difference can be made evident by carrying out the measurement in an invariant channel. For a sufficiently long measurement, the slope of the lines corresponding to the detected paths is apparent, and can be used to calculate the rate difference. 
Secondly, the fact that the delay resolution is of the order of $T_{c}$ is well known in the study of rake receivers for DS-SS systems [1, Sec. 14.5]. Two paths with a delay difference smaller than $T_{c}$ are not resolved by the receiver, and thus interfere, causing fading. This can be vividly illustrated with this measurement, as seen in Figure 3a.

\section{Application in a Course on Wireless Communications}

The techniques described can be applied in courses on wireless communications, either as laboratory work or as demonstrations performed by the teacher in the classroom. The former can bring greater student involvement, while the latter allows the teacher to more easily integrate measurements with exposition of the related theoretical content.

These measurement procedures have been used by the author in a course on mobile communications taught in the fifth year of the Telecommunication Engineering Degree of the Autonomous University of Madrid. Two or three short classroom demonstrations are usually employed, interlaced with the theoretical content. The experience has proven fruitful and rewarding. Students' interest during the measurements is high, and they are happy to participate when invited: for example, by displacing the microphone, or by operating the transmitting computer while the teacher controls the receiver. Questions asked by the teacher are important for encouraging the students to interpret what they are seeing, focusing their attention towards the key aspects that can be observed.

The additional features illustrated by the measurements are useful for relating multipath propagation with concepts learned from digital-transmission courses. This can broaden the vision of the subject and improve comprehension.

\section{Conclusions}

The use of sound waves to illustrate multipath propagation has been discussed. Three different techniques were proposed. The first characterized time dispersion, while the second dealt with time variation. The third technique described simultaneously gave information on both aspects of the channel, providing a full view of the time-variant impulse response, at the cost of reduced accuracy. In each case, the transmitted signal was suitably selected, and the receiver operations were discussed. Results were given that exemplified how the time-dispersion and time-variation aspects manifested in the measurements, and how they could be related to physical characteristics of the channel. Suggestions were given regarding application to courses on wireless communications.

\section{Acknowledgment}

The author thanks Dr. J. M. Hernando for his valuable guidance and help, which have made this study possible. In addition, he acknowledges Dr. A. Asensio for insightful discussions on pulsecompression techniques, and Dr. P. García for pointing out reference [4]. The author is also grateful to the Editor-in-Chief, Dr. W.
R. Stone, the Associate Editor, Dr. D. Kelley, and an anonymous reviewer for their positive and constructive comments.

\section{Appendix: Signal Design for Measuring the Impulse Response}

This appendix discusses signal design for the estimation of the impulse response. The two approaches introduced in Section 3 for time-invariant channels are analyzed in Sections 9.1 and 9.2, respectively. The conditions on the transmitted signal that yield a good estimation are then derived in Section 9.3. Based on this, the signal selection for a time-invariant channel is addressed in Section 9.4. Finally, the design is extended to time-variant channels in Section 9.5 .

\subsection{Design Based on Aperiodic Autocorrelation}

In the aperiodic design, $s(t)$ is expressed as

$$
s(t)=\sum_{i=0}^{M-1} S[i] g\left(t-i T_{c}\right),
$$

where $S[i]$ is a sequence of finite length $M$, defined for $n=0, \ldots, M-1$. The corresponding bandpass signal is

$$
\tilde{s}(t)=\operatorname{Re}\left[s(t) e^{\jmath 2 \pi f_{0} t}\right]
$$

and its quadrature phase-shifted version is defined as

$$
\tilde{s}^{\prime}(t)=\operatorname{Re}\left[s(t) e^{\jmath\left(2 \pi f_{0} t+\pi / 2\right)}\right]
$$

Let $\phi_{x}(\tau)=x^{*}(-\tau) * x(\tau)$ denote the autocorrelation function of a signal $x(t)$, and $\Phi_{X}[n]=X^{*}[-n] * X[n]$ is the aperiodic autocorrelation of a finite-length sequence $X[n]$. From $\tilde{r}(t)$, the receiver can compute

$$
\begin{aligned}
& y(\tau)=2 \tilde{s}(-\tau) * \tilde{r}(\tau), \\
& y^{\prime}(\tau)=2 \tilde{s}^{\prime}(-\tau) * \tilde{r}(\tau) .
\end{aligned}
$$

Neglecting double-frequency terms, which is valid if $f_{0} \gg 1 / T_{c}$, i.e., if the bandpass signal is "narrowband" [1, Sec. 4.1], it is easily seen that

$$
\begin{aligned}
& y(\tau)=\operatorname{Re}\left[h(\tau) * \phi_{s}^{*}(\tau)\right], \\
& y^{\prime}(\tau)=\operatorname{Im}\left[h(\tau) * \phi_{s}^{*}(\tau)\right] .
\end{aligned}
$$

Therefore, defining 


$$
z(\tau)=\sqrt{y^{2}(\tau)+y^{\prime 2}(\tau)},
$$

the following result is obtained:

$$
z(\tau)=\left|h(\tau) * \phi_{s}^{*}(\tau)\right| .
$$

\subsection{Design Based on Periodic Autocorrelation}

In this case,

$$
s(t)=\sum_{i=-m_{1}}^{M+m_{2}-1} S[i \bmod M] g\left(t-i T_{c}\right),
$$

where $S[i]$ is a sequence of finite length $M$. Initially, $m_{1}$ and $m_{2}$ are assumed to be $\infty$, i.e., $s(t)$ is periodic with period $M T_{c}$. Let $s_{p}(t)$ denote one period of $s(t)$, from $t=0$ to $M T_{c}$, and let $\tilde{s}_{p}(t), \tilde{s}_{p}^{\prime}(t)$ be the corresponding in-phase and quadrature-phaseshifted bandpass signals. The receiver computes

$$
\begin{aligned}
& y(\tau)=2 \tilde{s}_{p}(-\tau) * \tilde{r}(\tau), \\
& y^{\prime}(\tau)=2 \tilde{s}_{p}^{\prime}(-\tau) * \tilde{r}(\tau) .
\end{aligned}
$$

Let $\psi_{s_{p}}(\tau)=s_{p}^{*}(-\tau) \circledast s_{p}(\tau)$, where $\circledast$ denotes circular convolution. For the same conditions as in Section 9.1, Equation (5) gives

$$
\begin{aligned}
& y(\tau)=\operatorname{Re}\left[h(\tau) * \psi_{s_{\mathrm{p}}}^{*}(\tau)\right], \\
& y^{\prime}(\tau)=\operatorname{Im}\left[h(\tau) * \psi_{s_{\mathrm{p}}}^{*}(\tau)\right] .
\end{aligned}
$$

Since $s(\tau)$ is periodic with period $M T_{c}$, so are $\psi_{s_{p}}(\tau), y(\tau)$, and $y^{\prime}(\tau)$. This has two implications. First, the measurement has a delay ambiguity of $M T_{c}$ (this is analogous to range ambiguity in radar systems [3, Sec. 1.1]). To avoid this, $M$ should satisfy $M>\tau_{\max } / T_{c}$. Second, the receiver only needs to compute one period of $y(\tau)$ and $y^{\prime}(\tau)$. This will be assumed to be the period extending from $\tau=0$ to $M T_{c}$. As a result, the transmitted signal can be substituted for by a finite-length version, i.e., $m_{1}, m_{2}$ can be finite. Thus $s(t)$, rather than being a periodic signal, becomes a "cyclic extension" of $s_{p}(t)$. Since $h(\tau)$ vanishes for $\tau \notin\left[0, \tau_{\max }\right]$, it is easily seen that for any finite $m_{1}, m_{2}>\tau_{\max } / T_{c}$, the signals $y(\tau)$ and $y^{\prime}(\tau)$, restricted to $\tau \in\left[0, M T_{c}\right]$, coincide with those obtained for infinite $m_{1}, m_{2}$. (It is seen that compared with the aperiodic case, cyclically extending the signal transforms aperiodic correlation into periodic: this is analogous to the cyclic extension used in orthogonal frequency-division multiplexing, which transforms convolution into circular convolution [2, Sec. 3.4.4].) Consequently, if $m_{1}, m_{2}$ satisfy the stated condition, and if $z(\tau)$ is defined as in Equation (3), with $\tau$ restricted to the interval $\left[0, M T_{c}\right]$,

$$
z(\tau)=\left|h(\tau) * \psi_{s_{\mathrm{p}}}^{*}(\tau)\right| .
$$

\subsection{Conditions for Good Estimation}

In view of Equations (4) and (6), $z(\tau)$ can serve as an estimate of $|h(\tau)|, \tau \in\left[0, \tau_{\max }\right]$, in both the aperiodic and the periodic cases. The conditions for this estimation to be adequate are as follows. In the first case, since $h(\tau)$ appears after convolution with $\phi_{s}^{*}(\tau)$, the latter should resemble a narrow pulse. The function $\phi_{s}(\tau)$ can be expressed as

$$
\phi_{s}(\tau)=\sum_{i=1}^{M-1} \sum_{j=1}^{M-1} S^{*}[i] S[j] \phi_{g}\left[\tau+(i-j) T_{c}\right]
$$

from which

$$
\phi_{S}(\tau)=\sum_{k=-M+1}^{M-1} \Phi_{S}[k] \phi_{g}\left(\tau+k T_{c}\right)
$$

With the periodic approach, since $h(\tau)$ is convolved with the periodic function $\psi_{s_{p}}^{*}(\tau)$, a period of the latter should resemble a narrow pulse. Defining the periodic autocorrelation of $S[i]$ as

$$
\begin{gathered}
\Psi_{S}[n]=S^{*}[-n] \circledast S[n] \text {, the function } \psi_{S}(\tau) \text { is expressed as } \\
\psi_{s_{p}}(\tau)=\sum_{k=-\infty}^{\infty} \Psi_{S}[k] \phi_{g}\left(\tau+k T_{c}\right)
\end{gathered}
$$

Therefore, in view of Equations (7) or (8), two conditions have to be met for a good estimation:

1. $\phi_{g}(\tau)$ should be very narrow. This function determines the shape and width of the main lobe of $\phi_{s}(\tau)$ or $\psi_{s_{p}}(\tau)$, and thus the delay resolution.

2. $\Phi_{S}[n]$ or $\Psi_{S}[n]$ should be approximately zero for $n \in\{1, \ldots, M-1\}$. This is achieved by an appropriate choice of the sequence $S[i]$. This condition affects the sidelobe level of $\phi_{s}(\tau)$ or $\psi_{s_{p}}(\tau)$.

Condition 1 . basically reduces to selecting the smallest $T_{c}$ compatible with bandwidth restrictions. As for condition 2., with the aperiodic design, a convenient class of sequences is constituted by polyphase Barker sequences [6]. These have the form 


$$
S[i]=e^{\jmath 2 \pi \theta_{i} / J}, \theta_{i} \in\{0,1, \ldots, J-1\}
$$

for some $J \in \mathbb{N}$, and they achieve the maximum possible sidelobe level, equal to $M$, within all unit-modulus sequences. Polyphase Barker sequences are known up to $M=63[7,8]$ for values of $J$ that tend to increase with $M$. Binary Barker sequences $(J=2)$ are known for several values of $M$ up to 13 .

Regarding periodic autocorrelation, m-sequences [1, p. 433] are optimum. These are binary sequences, which exist for $M=2^{m}-1, m \in \mathbb{N}$, and the periodic autocorrelation of which, $\Psi_{S}[n]$, equals -1 for $n \bmod M \neq 0$, resulting in a $S L L=M$.

\subsection{Time-Dispersion Measurement}

For the time-dispersion measurement, the periodic design based on $\mathrm{m}$-sequences is preferred. The reason is that $\mathrm{m}$-sequences exist for greater lengths $M$ than polyphase Barker sequences, which gives larger sidelobe levels and $E_{s} / N_{0}$ in an invariant channel.

The non-coherent correlation, $z(\tau)$, given by Equation (6), is only useful for $\tau \in\left[0, M T_{c}\right]$, as seen in Section 9.2. Outside this interval, replicas of the central part are obtained as a result of the pattern signals $\tilde{s}_{p}(t)$ and $\tilde{s}_{p}^{\prime}(t)$ in Equation (5), partially matching the cyclic extensions present in $\tilde{r}(t)$. The representation should thus be adequately windowed. (The replicas have amplitudes $m_{1} / M$ and $m_{2} / M$ times that of the central part, respectively, and appear "noisier," as they have poorer autocorrelation properties.) The length of the window is $M T_{c}$, and its position can be manually adjusted.

\subsection{Time-Dispersion and Time-Variation Measurement}

A basic limitation in this case is that the channel must remain approximately invariant during the integration time; otherwise, the received signal will be "transparent" to the correlator, as it will not match the pattern signals. Note that frequency correction, as applied in Section 3 to compensate for clock-rate differences, is not useful here. This is because frequency deviations caused by movement are much more severe than clock-rate differences (for example, a radial velocity of $1 \mathrm{~m} / \mathrm{s}$ produces a Doppler shift equal to $0.34 \%$ of the carrier frequency), and are not necessarily constant during the integration period.

The low-pass equivalent transmitted signal is

$$
s(t)=\sum_{i=0}^{R-1} s_{r}\left(t-i T_{r}\right),
$$

according to Section 5.1. If the periodic approach is used, $s_{r}(t)$ is taken to be the signal $s_{p}(t)$ defined in Section 9.2, generated from an $\mathrm{m}$-sequence, and the integration time equals $T_{r}$. Since a cyclic shift of an m-sequence is also an m-sequence [1, Sec. 8.1.3], a resolution in $t$ equal to $T_{c}$ can be achieved, by correlating with different cyclic shifts of the sequence.

The problem with the periodic design is that $T_{r}$ determines both the maximum unambiguous delay, $\tau_{\max }$, and the maximum speed, $v_{\max }$. Namely, the delay should satisfy $\tau_{\max }<T_{r}$, and the condition that the channel remains approximately constant during $T_{r}$ is $v_{\max } \ll V /\left(f_{0} T_{r}\right)$. As a result, only channels with $v_{\max } \tau_{\max } \ll V / f_{0}$ can be measured. This condition is quite restrictive. As an example, for a moderate $\tau_{\max }$ of $0.2 \mathrm{~s}$ and $f_{0}=3.4 \mathrm{kHz}$, this gives $v_{\max } \ll 0.5 \mathrm{~m} / \mathrm{s}$. Numerical experiments show that for a binary sequence, an accumulated phase shift of $2 \pi / 3$ rad already causes noticeable degradation in the autocorrelation. $v_{\max }<0.17 \mathrm{~m} / \mathrm{s}$ was thus used in the example. The restriction could be alleviated by reducing $f_{0}$; however, this would degrade the delay resolution, because the chip rate must be significantly smaller than $f_{0}$, as seen in Section 9.1.

The referred drawback can be avoided by means of a design based on aperiodic autocorrelation. The low-pass equivalent transmitted signal, $s(t)$, is constructed from $s_{r}(t)$ as above. However, now the repeated signal, $s_{r}(t)$, has an active part $s_{a}(t)$ of length $T_{a}<T_{r}$, followed by a gap of length $T_{r}-T_{a}$. The correlation patterns, $\tilde{s}_{a}(t), \tilde{s}_{a}^{\prime}(t)$ are obtained from $s_{a}(t)$ as in Section 9.1. The integration time is $T_{a}$, and thus the maximum speed limitation is $v_{\max } \ll V /\left(f_{0} T_{a}\right)$, whereas the delay restriction is $\tau_{\max }<T_{r}$, as before. Both limitations can thus be controlled independently. In exchange for this flexibility, the resolution in $t$ is $T_{r}$ instead of $T_{c}$, as the technique of correlating with different cyclic shifts cannot be applied here. This can be solved by interpolating in $t$, provided that the channel variation during $T_{r}$ is reasonably smooth, i.e., that $v_{\max }$ is of the order of $V /\left(f_{0} T_{r}\right)$ or smaller (note that this is a weaker condition than requiring that the channel be approximately constant during $T_{r}$ ). Otherwise, the interpolation will miss some of the channel variation, giving a smoothed, possibly aliased, version.

For a signal $\tilde{s}_{a}(t)$ consisting of $M$ chips, the speed limitation described determines the usable values of $M$. For example, taking $T_{c}=1 \mathrm{~ms}, f_{0}=3 \mathrm{kHz}$, and $v_{\max }=1 \mathrm{~m} / \mathrm{s}$ as typical values requires $M \ll 113$. In view of this, the fact that polyphase Barker sequences are known only for $M \leq 63$ is of no importance. In addition, numerical computation shows that the autocorrelation properties of these sequences are more sensitive to channel changes within $T_{a}$ as the alphabet size, $J$, increases. Since with currently known sequences, $J$ grows rapidly with $M$ (for example, $M>15$ requires $J \geq 6$ : see [6] and the references therein), the binary sequence of length $M=13$ or the six-phase sequence of $M=18$ are seen as good choices, with $\mathrm{SLL}=22.3 \mathrm{~dB}$ or $25.1 \mathrm{~dB}$, respectively. Of course, these small lengths give modest values of $E_{s} / N_{0}$; nevertheless, noise is well below autocorrelation secondary lobes for moderate volume levels at the transmitter.

Considering the above, the aperiodic design is chosen, with polyphase (or binary) Barker sequences of small lengths. The receiver computes $z(\tau)$ as in Equations (2) and (3), except that $\tilde{s}(t), \tilde{s}^{\prime}(t)$ are replaced by $\tilde{s}_{a}(t), \tilde{s}_{a}^{\prime}(t)$. This gives a result 
analogous to that in Section 9.1, only that repeats every $T_{r}$. Each repetition corresponds to a "snapshot" of the channel, i.e., an estimation of $\left|h\left(t_{i}, \tau\right)\right|, t_{i+1}=t_{i}+T_{r}$, and there is a delay ambiguity of $T_{r}$.

\section{References}

1. J. G. Proakis, Digital Communications, Fourth Edition, New York, McGraw-Hill, 2001.

2. D. Tse and P. Viswanath, Fundamentals of Wireless Communication, Cambridge, Cambridge University Press, 2005.

3. M. I. Skolnik (ed.), Introduction to Radar Systems, Second Edition, New York, McGraw-Hill, 1980.

4. J. Watkinson, The Art of Digital Audio, Third Edition, Oxford, UK, Focal Press, 2001.

5. J. M. Hernando and F. Pérez-Fontán, Introduction to Mobile Communications Engineering, Norwood, MA, Artech House, 1999.

6. J. Jedwab, "What Can be Used Instead of a Barker Sequence?," Contemporary Mathematics, 461, 2008, pp. 153-178.

7. P. Borwein and R. Ferguson, "Polyphase Sequences with Low Autocorrelation," IEEE Transactions on Information Theory, 51, 4, April 2005, pp. 1564-1567.

8. A. R. Brenner, "Polyphase Barker Sequences Up to Length 45 with Small Alphabets," Electronics Letters, 34, 16, August 1998, pp. 1576-1577. (4i) 